Journal of

Synchrotron

Radiation

ISSN 1600-5775

Received 15 October 2013

Accepted 14 February 2014

\section{High-efficiency zone-plate optics for multi-keV $X$-ray focusing}

\author{
Istvan Mohacsi, ${ }^{\mathrm{a}, \mathrm{b} *}$ Petri Karvinen, ${ }^{\mathrm{a}}$ Ismo Vartiainen, ${ }^{\mathrm{a}}$ Vitaliy A. Guzenko, ${ }^{\mathrm{a}}$ \\ Andrea Somogyi, ${ }^{b}$ Cameron M. Kewish, ${ }^{b}$ Pascal Mercere ${ }^{b}$ and Christian David ${ }^{a}$ \\ 'Paul Scherrer Institut, 5232 Villigen, Switzerland, and 'bynchrotron SOLEIL, L'Orme des Merisiers, \\ Saint-Aubin, 91192 Gif-sur-Yvette, France. *E-mail: istvan.mohacsi@psi.ch
}

\begin{abstract}
High-efficiency nanofocusing of hard X-rays using stacked multilevel Fresnel zone plates with a smallest zone width of $200 \mathrm{~nm}$ is demonstrated. The approach is to approximate the ideal parabolic lens profile with two-, three-, four- and sixlevel zone plates. By stacking binary and three-level zone plates with an additional binary zone plate, the number of levels in the optical transmission function was doubled, resulting in four- and six-level profiles, respectively. Efficiencies up to $53.7 \%$ focusing were experimentally obtained with $6.5 \mathrm{keV}$ photons using a compact alignment apparatus based on piezoelectric actuators. The measurements have also been compared with numerical simulations to study the misalignment of the two zone plates.
\end{abstract}

Keywords: Fresnel zone plates; zone-plate stacking; X-ray nanofocusing.

\section{Introduction}

Most scanning X-ray microscopy methods at synchrotron radiation facilities rely on focusing $\mathrm{X}$-ray optics to obtain high spatial resolution (Kaulich et al., 2011). Furthermore, the flux density in the focal spot may reach up to $10^{5}-10^{6}$ times that of the incident beam's density. The gain in the available photon flux to user experiments improves the measurement speed and analytical sensitivity and allows more efficient use of the available beam time. Amongst the different focusing methods, Kirkpatrick-Baez (KB) mirror systems (Kirkpatrick \& Baez, 1948) have gained large popularity due to their high focusing efficiency, small achievable spot size and achromatic behavior. However, these systems are bulky and can be challenging to align, especially when a large aperture size (hundreds of micrometers) is required. Conventional binary diffractive X-ray optics, including Fresnel zone plates (Soret, 1875), serve as compact easy-to-align focusing elements that allow for a straight optical axis and aperture sizes up to millimeters. Their main drawback is their relatively low efficiency, fundamentally limiting the intensity of the first-order focus up to $40.5 \%$ of the incident intensity for an ideal non-absorbing binary zone plate. The symmetric cross section of the binary line profile leads to the symmetry of the focusing positive and the defocusing negative diffraction orders. Consequently, half of the intensity is diffracted into the divergent orders and is blocked by the order-sorting aperture. As the phase shift is directly proportional to the structure height, the fabrication of structures with the optimal phase shift requires up to several micrometers high structures in the multi-keV energy range. This becomes an issue when a small spot size is needed, as the numerical aperture and thus the diffraction-limited spot size is defined by the smallest zone width (Kirz, 1974). Therefore, the fabrication of high-resolution diffractive X-ray optics requires the fabrication of high-aspect-ratio nanostructures.

A method has been proposed (Maser et al., 1999) to increase the structure height of Fresnel zone plates by stacking two identical binary zone plates on the same optical axis in each others optical near-field, to act as a single zone plate with added zone heights. This approach indeed relaxes the required aspect ratios; however, it can still only provide binary zone plates and hence it is limited by the equal positive and negative diffraction orders. In the following paragraph we will briefly discuss that, by using asymmetric multilevel structures, the focusing efficiency of Fresnel zone plates can break the fundamental limit of binary zone plates.

Originally, refractive Fresnel lenses were developed as large aperture and short focal length lenses, specifically designed for lighthouses (Watson \& Scott, 2003). The aim was to reduce the weight of the required glass in comparison with traditional lenses. Using less lens material is also important in the X-ray range, although for a different reason: the applied high phase shifter materials are not completely transparent in the X-ray range, resulting in absorption losses within the lens material. Therefore, X-ray lenses must minimize the optical path within the lens due to absorption in the lens material. As Fresnel zone plate lenses in the $\mathrm{X}$-ray range have small numerical apertures, within the paraxial approximation, they must provide a similar phase shift profile as their equivalent refractive counterpart with parabolic phase shift profile (Fig. 1a). Since in the case of a diffractive lens the complex phase of the electromagnetic radiation has a redundant $2 \pi$ 
a)

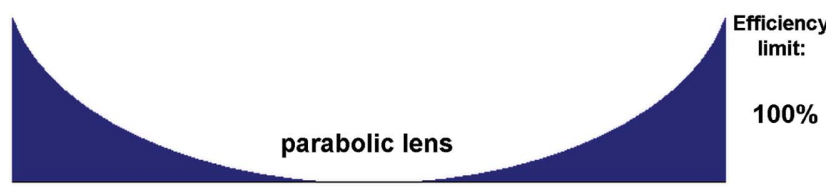

wrapped parabolic lens

b) $M N / 1 / 1 / 1 / 100 \%$

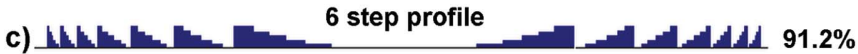

4 step profile

d) thath the $81.1 \%$

3 step profile

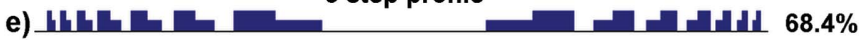

2 step (binary) profile

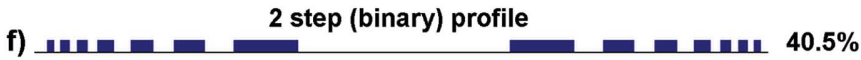

Figure 1

Fresnel zone plate lenses can be deducted from the ideal lens with parabolic phase-shift profile $(a)$, by wrapping the phase profile modulo $2 \pi$ to minimize the absorption in the lens material $(b)$. Owing to manufacturing difficulties of the continuous blazed profile, multilevel zone plates are the discrete approximations of the phase-wrapped lens ( $c-$ $e)$. In this respect the binary Fresnel zone plate represents the most basic two-level approximation $(f)$. The fundamental limit of diffraction efficiency increases with better approximation (right).

periodicity, lenses can be wrapped modulo $2 \pi$, while giving the same wavefront resulting in a similar design as the Fresnel lens (Fig. 1b).

However, the nanofabrication of the required quasi-triangular structures with small lateral dimensions is impossible using conventional lithographic tools. Therefore, in practice, X-ray lenses are designed to be the discrete ('staircase') approximation of the phase-wrapped ideal lens profile with a given number of steps (Figs. $1 c-1 f$ ). In these terms, the binary zone plates represent the most basic two-level approximations and, once the $\pi$ phase shift is reached, the approximation can only be improved by adding additional levels. Such multilevel zone plates have been previously demonstrated (DiFabrizio et al., 1999) to reach diffraction efficiencies up to $55 \%$, but this required the compromise of $1 \mu \mathrm{m}$ effective smallest zone width (the smallest zone width of multilevel zone plates is the half-pitch).

The concepts of multilevel and stacked zone plates have been combined (Chen et al., 2009) by stacking two different binary zone plates, a 'coarse' zone plate with $\pi$ phase shift and a double density 'fine' zone plate with $\pi / 2$ phase shift in each others optical near-field. This geometry provides an effective four-level staircase profile (Fig. 2a), resulting in a theoretical diffraction efficiency of up to $81 \%$ for an ideal non-absorbing stack. However, the pioneering authors only achieved a diffraction efficiency of $29 \%$, which is even achievable by binary zone plates.

In this paper, we demonstrate the viability of the concept to use blazed stacking (Fig. $2 a$ ) in order to surpass the limitations of binary zone plates. Furthermore, we expand the concept by showing that a suitable binary dense zone plate can be stacked with either a binary or a multilevel coarse zone plate in order to double the effective number of steps and obtain higher focusing efficiency. For any $n$-level zone plate, the width and height of each step should correspond to $\lambda / n$ path length

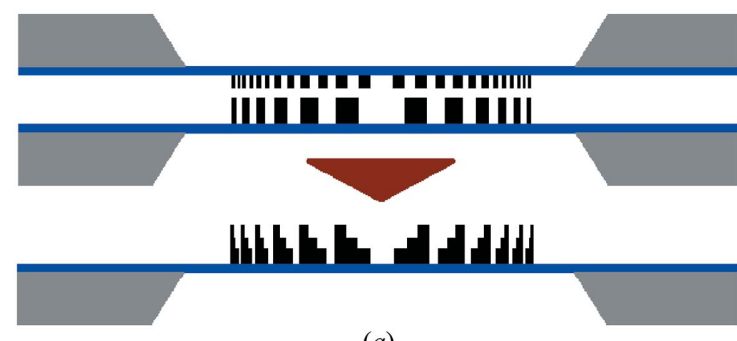

(a)

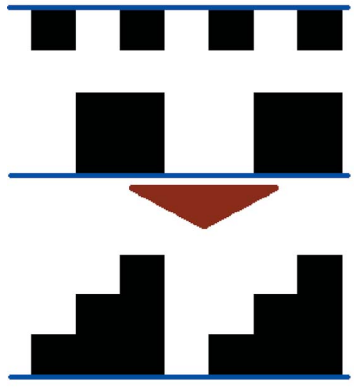

(b)

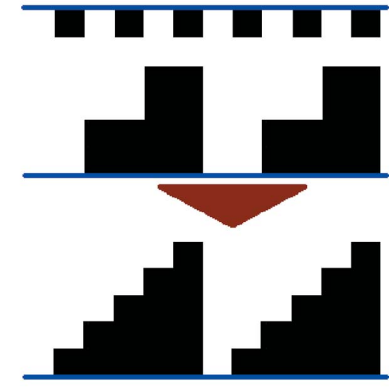

(c)
Figure 2

(a) The stacking of two binary zone plates in order to obtain an effective four-level profile. (b) Cross section of a zone in the case of stacking two binary zone plates to obtain a four-level profile and $(c)$ stacking a threelevel zone plate with a binary zone plate to obtain an effective six-level profile. The zone width is always the half-pitch, regardless of the number of steps.

difference to the focus. A good approximation in the nonabsorbing and weakly absorbing case is to subdivide the $\delta$ pitch into $n$ equally wide steps of $\delta / n$ width and $2 \pi / n$ phase shift (Nöhammer et al., 2003). The resulting zone placement error rapidly decreases with the increasing number of zones. This approximation gives a binary zone plate when $n=2$ and a multilevel zone plate if $n>2$. The smallest zone width is always determined as the smallest half-pitch. Any $n$-level single zone plate can be stacked with an $n$-times denser binary zone plate of $\pi / n$ phase shift to double the effective number of steps for improved focusing efficiency. In order to demonstrate the concept, we designed two experimental set-ups: the first set-up was based on the stacking of two different binary zone plates to create an effective four-level profile (Fig. $2 c$ ), while the second set-up used a monolithic three-level zone plate as a coarse zone plate combined with a triple-density binary zone plate to double the number of steps and achieve an effective six-level profile (Fig. 2c).

\section{Sample fabrication}

The aim of our experiment was to provide high-efficiency focusing optics for X-rays in the $5-8.5 \mathrm{keV}$ energy range. In this range nickel was chosen as a low-absorbing strong-phaseshifting material for fabrication of up to $500 \mu \mathrm{m}$-diameter Fresnel zone plates with effective outermost zone width of $200 \mathrm{~nm}$. Owing to the low, but not negligible, absorption the fundamental efficiency limit of the fabricated zone plates is lower than that of their ideal counterparts. Nominally at $6.5 \mathrm{keV}$ the efficiency of nickel zone plates is limited to $37.1 \%$ for binary, $60.7 \%$ for three-level, $70.9 \%$ for four-level and 
$78.5 \%$ for six-level profiles. The zone profiles were not optimized for absorption in the lens material and the zone placement of the three-step zone plates was approximated with equal steps due to the difference being below the accuracy of the manufacturing process.

Our fabrication process was based on the previously described fabrication of high-aspect-ratio gold nanostructures (Gorelick et al., 2010). This method is based on filling an electron-beam-written PMMA mold with metallic nanostructures using electroplating. The zone plates were prepared on $250 \mathrm{~nm}$-thick $\mathrm{Si}_{3} \mathrm{~N}_{4}$ membrane windows on $\mathrm{Si}$ frames with $20 \mathrm{~nm}$ gold plating base and a PMMA resist layer on top. The zone-plate patterns were exposed into the PMMA resist layer with a $100 \mathrm{keV}$ Vistec EBPG 5000+ electron-beam writer. The patterns were prepared by an optimized code (Guzenko et al., 2010), which greatly reduces the overheads in the exposure time due to data fracturing and adds radial support structures to enhance mold stability. The zone-plate patterns were arranged in order to fit within a single write field of the electron-beam writer; only beam deflection (i.e. no stage movement) was used during the exposure of the zone plates. Since the sidewall slope plays an important role in zone-plate performance, exposure parameters in terms of dose and line shrinkage were carefully optimized to obtain the correct zone cross sections and to avoid non-vertical sidewalls due to scattering processes during the e-beam exposure.

The exposed samples were developed in a mixture of 2propanol and water ( $7: 3$ by volume) cooled to $275 \mathrm{~K}$ for increased contrast and achievable aspect ratios (Shazia et al., 2002). The PMMA mold was filled with electroplated nickel using pulsed plating to obtain uniform structure height (Lindblom et al., 2006). For the three-level monolithic zone plates, we exposed additional alignment markers together with the first layer of zone-plate patterns. After developing and electroplating the first layer, we spin-coated an additional layer of PMMA over the filled mold and precisely aligned the second exposure over the first layer using the exposed alignment markers. After development the trenches were cleaned by oxygen plasma etching and filled with electroplated nickel. The PMMA mold was subsequently removed from all samples via ashing in oxygen plasma. Samples were pre-characterized by optical and scanning electron microscopy (SEM) in order to select the most promising candidates for X-ray analysis (Fig. 3).

\section{Simulations}

We developed a numerical model to simulate stacking and alignment specific errors of zone-plate stacks to provide a better understanding of our experimental measurements. Previous simulations on the stacking of Fresnel zone plates were calculated in the case of circular symmetry (VilaComamala et al., 2013). To model our measurements taking into account misalignment and aberrations that break the circular symmetry, we developed an advanced model based on the free-space propagation of a two-dimensional wavefield using the angular spectrum method (Ersoy, 2006). The simu-

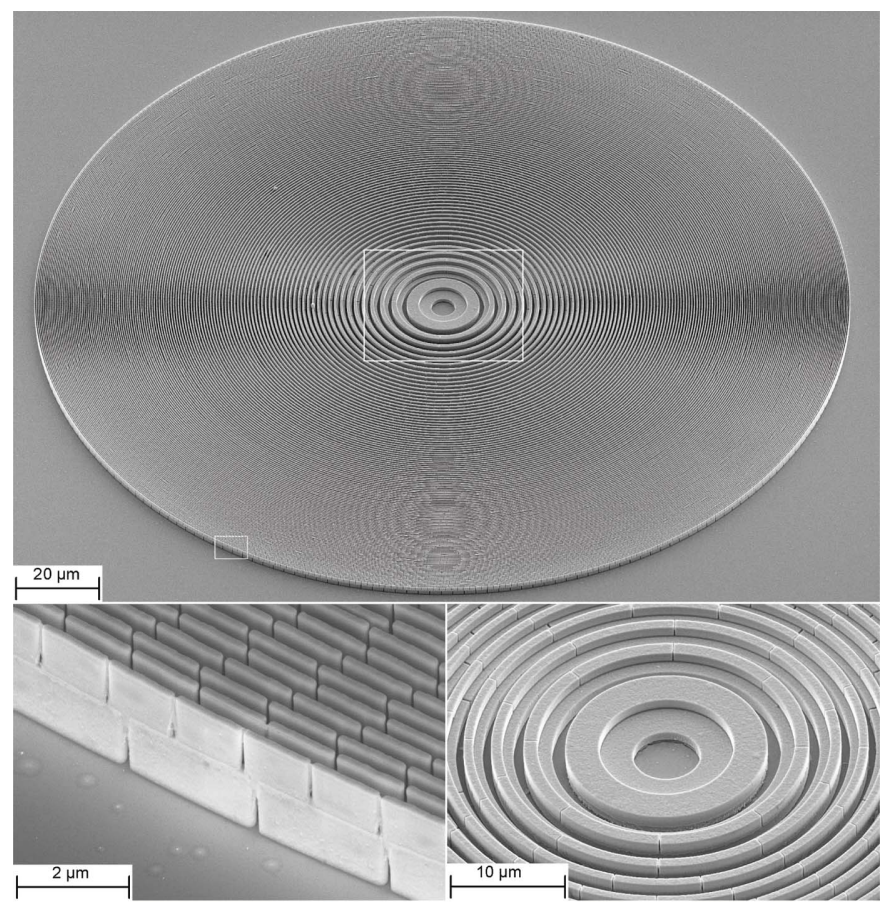

\section{Figure 3}

An example of a three-level nickel zone plate with $200 \mu \mathrm{m}$ diameter and effective $200 \mathrm{~nm}$ smallest zone width (top). The marked regions can be seen below with higher magnification, showing the individual steps of $2 / 3$ and $1 / 3$ fill factor at the center and at the edge of the zone plate. The effective smallest zone width is the half-period of $200 \mathrm{~nm}$.

lations were run on a desktop computer using GPGPU acceleration for increased speed. The presented simulations were all performed for nickel zone-plate stacks at $6.5 \mathrm{keV}$ photon energy. The stacking distance between the two zone plates was assumed to be $5 \mu \mathrm{m}$ as a typical value for our experimental set-up.

Our calculations show that zone-plate stacks indeed provide the best efficiency when they are on the same optical axis, but that their alignment around the common optical axis is tolerant to small misalignment within about one-third of the smallest zone width of the stack (Fig. 4). For larger misalignment the focal spot splits into multiple peaks and the efficiency drops to an approximately constant level with slight concentric rings around the alignment peak. The size of the focal spot follows a similar trend as the focusing efficiency; therefore, small misalignment does not increase the spot size significantly. It is also notable that the measurable focusing efficiency of finite-thickness zone plates in the vicinity of the focal spot (see experimental set-up in Fig. 5) is slightly lower than the predicted diffraction efficiency using the simple grating model.

In practice, the prepared zone-plate patterns are slightly distorted due to imperfections of the electron-beam writer, unwanted tilt of the substrate during the writing process, or by stress in the applied resist layers. These prevent the simultaneous alignment of the whole zone-plate area during stacking and reduce the focusing efficiency of the zone-plate stack, while increasing the tolerance to small misalignments around the optical axis. 


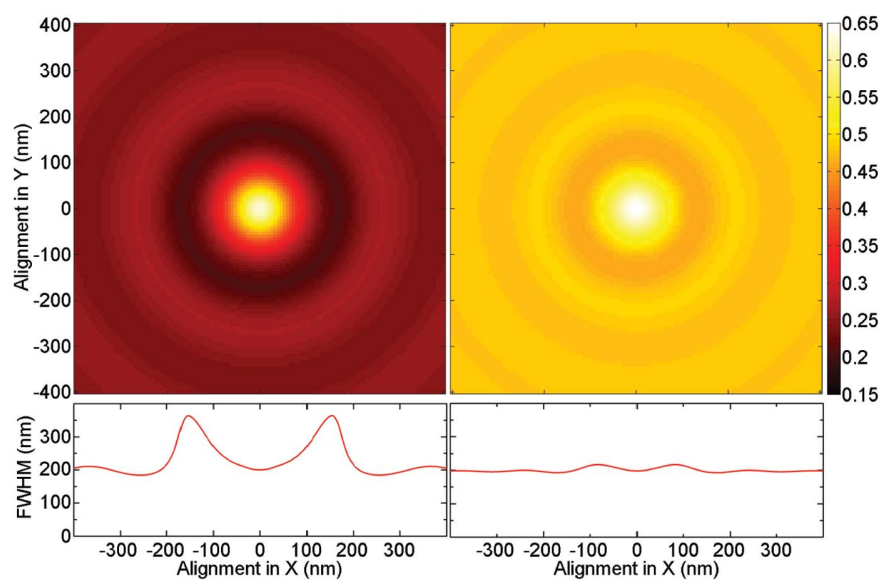

Figure 4

Simulated efficiency versus misalignment for the described four-level (top left) and six-level (top right) Fresnel zone plate stacks, with smallest zone width of $200 \mathrm{~nm}$. The central peak is tolerant for small misalignments within one-third of the smallest zone width, while the focusing efficiency drops greatly for larger misalignments. The full width at half-maximum (FWHM) of the spot size can be seen below, showing similar tolerance for misalignment as the focusing efficiency. The oscillations of the spot size correspond to splitting into multiple spots.

\section{Experimental methods and measurements}

The selected sets of zone plates were tested at the cSAXS beamline of the Swiss Light Source and at the Metrology beamline of the Synchrotron SOLEIL to characterize their performance as individual and as stacked optical elements. We used $6.5 \mathrm{keV}$ photon energy for all presented measurements (wavelength of $0.19 \mathrm{~nm}$ ).

In our experimental set-up to measure the diffraction efficiency of individual zone plates, the generally used first diffraction order was selected using a $20 \mu \mathrm{m}$ pinhole as an order-selecting aperture (OSA) and the flux was recorded with a large-area X-ray photodiode detector further downstream. We scanned the OSA across the focal plane, thereby recording the background (zeroth order, air scattering) and recording an intensity peak, when the focal spot was within the OSA. The measured intensity was converted to efficiency by subtracting the background and normalizing it with the illuminating intensity measured through a reference pinhole of equal size as the zone plate. We could not explicitly measure the diffraction-limited spot size of our lenses due to the incoherent illumination of the large $(200 \mu \mathrm{m}$ - and $500 \mu \mathrm{m}$ diameter) zone-plate aperture limiting our resolution to the demagnified source size. The best 'coarse' zone plates were selected for stacking based on their first-order focusing efficiency. The 'fine' zone plates did not have a similar obvious performance indicator, as their efficiency is heavily dependent on the structure height aside of defects and the fill factor of the zones; therefore they were chosen based on the prior SEM inspection.

Using this method we characterized monolithic binary and three-level zone plates. From the characterized $500 \mu \mathrm{m}$ diameter binary zone plates the best lens with $31.0 \%$ focusing efficiency was selected for stacking with a dense binary zone plate. In the case of the $200 \mu \mathrm{m}$-diameter three-level zone

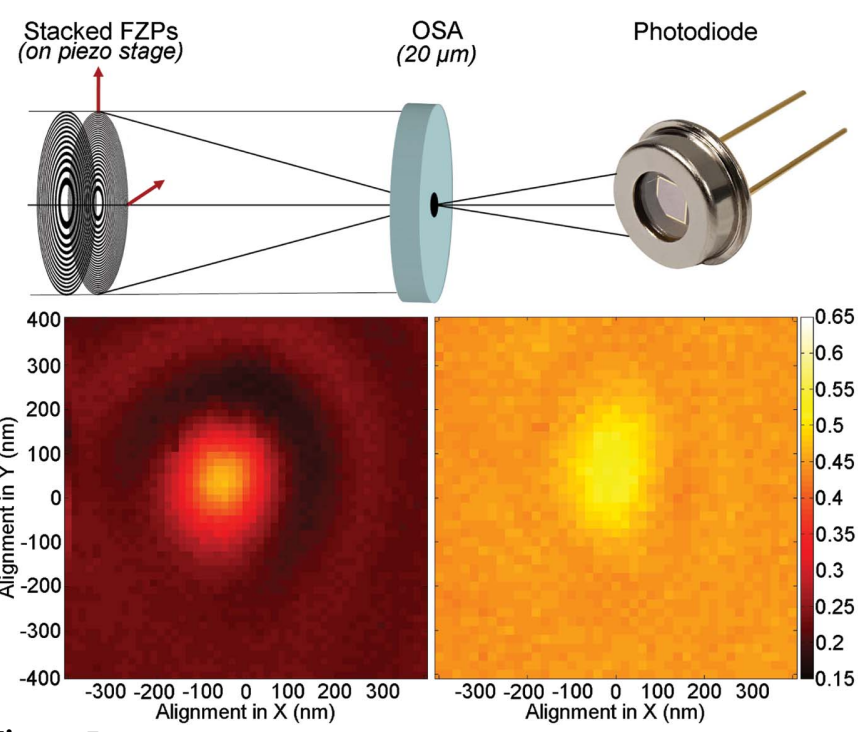

Figure 5

Experimental set-up for stacked zone-plate efficiency measurements (top). The dense zone plate was scanned around the common optical axis, while the diffraction efficiency in the first order was recorded. Measured diffraction efficiencies at small misalignments for effective four-level (bottom left) and six-level (bottom right) stacked zone plates with $47.1 \%$ and $53.7 \%$ diffraction efficiency in their best alignment.

plates the best result was $40.4 \%$ focusing efficiency. Since the fundamental limit of binary nickel zone plates is $37.1 \%$, this already demonstrates the advantage of using multilevel zone plates.

For the stacking experiments we used a special set-up aimed at the flexible stacking of our lenses (Fig. 5a). To achieve the required alignment precision a two-step alignment procedure was used. The coarse zone plate was mounted on a fixed stage with mechanical alignment allowing a large movement range. The fine zone plate was mounted on an $X Y Z$ piezo stage with a movement range of $100 \mu \mathrm{m}$. The mechanical pre-alignment was performed offline with the aid of an optical microscope in order to bring the two zone plates within the piezo range. We also eliminated any excessive tilt of the two zone plates by adjusting them parallel to the microscope's focal plane. The final alignment was made in the X-ray beam by scanning the alignment piezo while monitoring the diode signal. By normalizing these data, we can plot the diffraction efficiency of the stack as a function of alignment, showing that the efficiency peaks around the common optical axis (in agreement with simulations). The diffraction efficiency was determined as the height of this peak, corresponding to the best alignment of the two zone plates (see Fig. 5, bottom). We measured diffraction efficiencies up to $47.1 \%$ using binary zone plates to create a four-level profile and up to $53.7 \%$ when combining a three-level with a binary zone plate to create an effective six-level profile. We have also experimentally verified that, although the optimal energy is $6.5 \mathrm{keV}$, the focusing efficiency of the six-level stack stays beyond $40 \%$ in the whole 6-8 $\mathrm{keV}$ range.

It is interesting to note that the patterns are slightly distorted from their expected circular symmetry, suggesting that the zone plates are slightly distorted from their ideal 


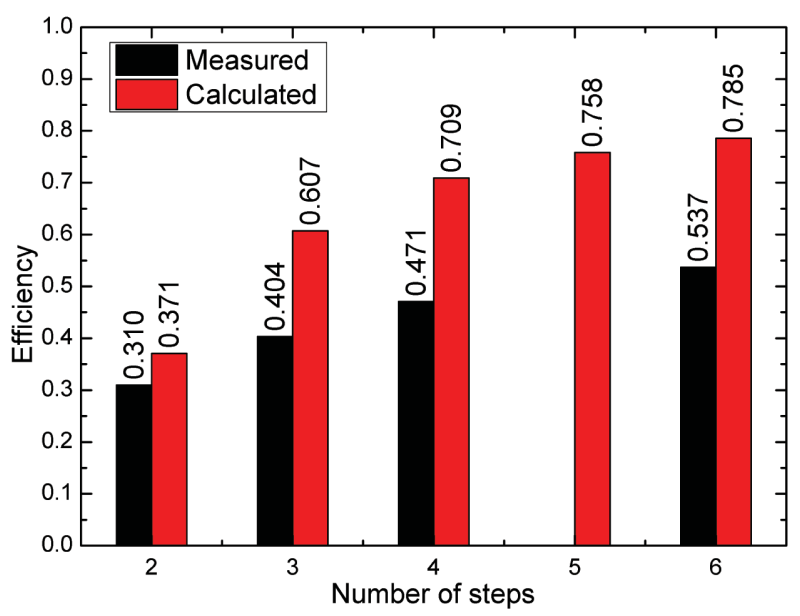

Figure 6

Calculated and measured focusing efficiencies as a function of the number of levels for nickel zone plates at $6.5 \mathrm{keV}$. The discrepancy is caused by manufacturing errors and pattern distortions. The two curves follow the same trend; the addition of further levels results in a smaller relative gain as the number of levels increases.

circular shape. This can be caused either by stress in the resist layer or in the plated nickel, tilt of the substrate during the ebeam writing, or distortions in the e-beam writers write field. The distortions cause negligible aberrations in the wavefront of a single zone plate, but in the case of stacking it prevents the perfect overlap of the two zone plates in a stack, reducing the achievable efficiency. This is discussed comprehensively elsewhere (Mohacsi et al., 2013).

When comparing the measured efficiencies with the theoretical values it becomes evident that, while the binary zone plate achieved over $80 \%$ of the calculated value, the multilevel and stacked efficiencies are only $65-70 \%$ of the theoretical values (Fig. 6). The differences have two major contributors: on one part some degree of manufacturing errors are still present even in optimized structures and can add up during stacking; the second and possibly the dominant cause is the distortions preventing the perfect overlap of the stack. Nevertheless, the addition of further levels to the zone-plate stack helped to improve the efficiency, but the gain of adding further levels becomes lower.

\section{Conclusion}

We have successfully combined multilevel zone plates with zone-plate stacking to achieve both high-resolution and highefficiency nanofocusing at $6.5 \mathrm{keV}$ photon energy. By stacking two binary zone plates, forming an effective four-level profile, we achieved $47.1 \%$ diffraction efficiency over very large $500 \mu \mathrm{m}$ aperture size, while having a $200 \mathrm{~nm}$ smallest zone width, corresponding to a diffraction-limited spot size of $250 \mathrm{~nm}$. With the achieved high resolution and high efficiency over a large aperture size, the flux density at the focal spot is expected to be $>10^{6}$ times more intense than the illuminating beam. We also moved a step forward by generalizing zoneplate stacking into the step-doubling of multilevel zone plates. We have experimentally verified the concept by stacking a three-level zone plate with a binary zone plate, forming an effective six-level profile and achieving $53.7 \%$ diffraction efficiency over a $200 \mu \mathrm{m}$ aperture, while keeping the resolution unchanged. These properties, together with the advantage of a compact size and maintaining a straight optical axis, can make stacked Fresnel zone plates an attractive alternative of $\mathrm{KB}$ mirrors in some applications.

The authors are grateful for A. Lücke, B. Haas, E. Deckardt and J. Lehmann for their assistance during sample preparation, and for Ana Diaz for her help during related measurements, revealing initial fabrication errors of our early lenses. This project was carried out as a joint effort between the Laboratory for Micro- and Nanotechnology (PSI) and the Nanoscopium beamline of Synchrotron SOLEIL. Part of this work was performed at the Swiss Light Source, Villigen, Switzerland. We acknowledge SOLEIL for provision of synchrotron radiation facilities (proposal ID 99130006) and we would like to thank the beamline staff for using the Metrology beamline. The research leading to these results has received funding from the European Community's Seventh Framework Programme (FP7/20072013) under grant agreement No. 290605 (PSI-FELLOW/COFUND).

\section{References}

Chen, S., Lyon, A., Kirz, J., Seshadri, S., Feng, Y., Feser, M., Sassolini, S., Duewer, F., Zeng, X. \& Huang, C. (2009). Proc. SPIE, 7448, 74480D.

DiFabrizio, E., Romanato, F., Gentili, M., Cabrini, S., Kaulich, B., Susin, J. \& Barrett, R. (1999). Nature (London), 401, 895-898.

Ersoy, O. K. (2006). Diffraction, Fourier Optics and Imaging. New York: Wiley.

Gorelick, S., Guzenko, V. A., Vila-Comamala, J. \& David, C. (2010). Nanotechnology, 21, 295303.

Guzenko, V. A., Romijn, J., Vila-Comamala, J., Gorelick, S. \& David, C. (2010). AIP Conf. Proc. 1365, 92-95.

Kaulich, B., Thibault, P., Gianoncelli, A. \& Kiskinova, M. (2011). J. Phys. Condens. Matter, 23, 083002.

Kirkpatrick, P. \& Baez, A. V. (1948). J. Opt. Soc. Am. 38, 766-774.

Kirz, J. (1974). J. Opt. Soc. Am. 20, 301-309.

Lindblom, M., Hertz, H. M. \& Holmberg, A. (2006). J. Vac. Sci. Technol. B, 24, 2848.

Maser, J., Lai, B., Yun, W., Shastri, S. D., Cai, Z., Rodrigues, W., Xua, S. \& Trackhtenberg, E. (1999). Proc. SPIE, 4783, 74-81.

Mohacsi, I., Karvinen, P., Vartiainen, I. J., Diaz, A., Somogyi, A., Kewish, C. M., Mercère, P. \& David, C. (2013). Proc. SPIE, 8851, $88510 \mathrm{Z}$

Nöhammer, B., David, C., Gobrecht, J. \& Herzig, H. P. (2003). Opt. Lett. 28, 1087-1089.

Shazia, Y., Hasko, D. \& Ahmed, H. (2002). Microelectron. Eng. 61-62, 745-753.

Soret, J. L. (1875). Arch. Sci. Phys. Nat. 52, 320.

Vila-Comamala, J., Wojcik, M., Diaz, A., Guizar-Sicairos, M., Kewish, C. M., Wang, S. \& David, C. (2013). J. Synchrotron Rad. 20, 397404.

Watson, M. D. \& Scott, S. M. (2003). Encycl. Opt. Eng. 1, 616-627. 